\title{
Microfluidic diagnostic technologies for global public health
}

\author{
Paul Yager ${ }^{1}$, Thayne Edwards ${ }^{1}$, Elain Fu', Kristen Helton ${ }^{1}$, Kjell Nelson ${ }^{1}$, Milton R. Tam² \& Bernhard H. Weigl ${ }^{3}$
}

\begin{abstract}
The developing world does not have access to many of the best medical diagnostic technologies; they were designed for air-conditioned laboratories, refrigerated storage of chemicals, a constant supply of calibrators and reagents, stable electrical power, highly trained personnel and rapid transportation of samples. Microfluidic systems allow miniaturization and integration of complex functions, which could move sophisticated diagnostic tools out of the developed-world laboratory. These systems must be inexpensive, but also accurate, reliable, rugged and well suited to the medical and social contexts of the developing world.
\end{abstract}

Microfluidic systems can be designed to obtain and process measurements from small volumes of complex fluids with efficiency and speed, and without the need for an expert operator; this unique set of capabilities is precisely what is needed to create portable point-of-care (POC) medical diagnostic systems ${ }^{1,2}$. Fortunately for the microfluidics field, the military has always had a need to practise medicine in challenging and resource-limited environments, and so has long been trying to acquire robust medical technologies that add an absolute minimum to the burden of those people and machines transporting them. It was for this reason that microfluidics research in the United States was given a great boost in the 1990s by funding from the US Defense Advanced Research Projects Agency (DARPA). The technologies developed with DARPA's support (for examples, see www.darpa.mil/MTO/mFlumes) have the characteristics needed for delivering appropriate medical diagnostics to the world's poorest people. Today, the potential of microfluidic technologies to enhance the decentralization of medical testing is becoming accepted as one element in the next stage in the evolution of healthcare. Thanks to an upsurge in interest in (and funding of) healthcare in the developing world, combined with a slow pace of change in the developed world, this new microfluidic diagnostic technology (MDT) may be adopted first for civilian healthcare in the developing world. Some initial steps towards designing appropriate microfluidic diagnostic systems are described here.

\section{Overview of global health issues}

In recent decades, substantial progress has been made in public health, but this progress has not been equal in developed and developing countries. The benefits and services that we in the West take for granted are often inadequate or lacking in many developing countries $^{3,4}$. For example, of the world's population of 6.1 billion people, 3 billion lack basic sanitation, 2 billion do not have access to electricity and more than 1 billion lack basic healthcare services and clean drinking water ${ }^{5,6}$. For every public health triumph such as the eradication of smallpox, other infectious diseases such as tuberculosis and malaria have re-emerged, accompanied by new diseases such as HIV/AIDS (Fig. 1). More than half the deaths in the poorest countries are the result of infectious diseases (compared with less than $5 \%$ in the richest $)^{7,8}$. Global health, poverty and development are interdependent. Endemic poverty is a significant impediment to improving health ${ }^{9}$. One billion, or half of the world's children, live

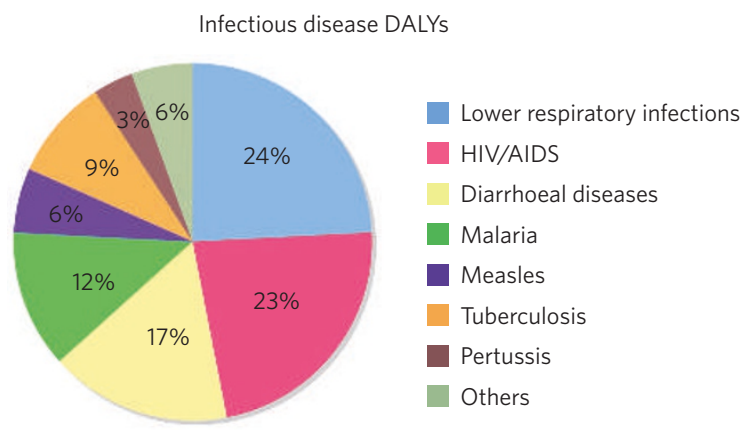

Figure 1 | Disability-adjusted life years (DALYs) for infectious and parasitic diseases. To properly reflect the full impact of a disease, disease burdens can be measured in DALYs by adding the years of life lost by a person's premature death to the time lived with a disability. Infectious and parasitic diseases accounted for almost $30 \%$ of all DALYs and 15 million deaths each year worldwide. Shown are the infectious and parasitic diseases responsible for the DALYs in 2005 (figures from the US Centers for Disease Control and Prevention).

in poverty. Health indicators for mothers, newborns and children in many of the poorest countries have remained the same or have even declined in recent years ${ }^{10}$.

Until recently, diagnostic tests were not routinely developed primarily for developing-country markets, although two exceptions are the Program for Appropriate Technology in Health (PATH) HealthTech initiative, discussed below, and Helen Lee's diagnostics development programme at the University of Cambridge, UK. Led by the Bill \& Melinda Gates Foundation, initial funding has been provided to search for innovative technologies and solutions, including development of new in vitro diagnostic tests and test platforms, for the world's neglected diseases ${ }^{11}$. The US National Institute of Allergy and Infectious Disease is also funding an in vitro diagnostics development programme for biodefence to address these issues ${ }^{12}$. Three additional initiatives that focus on the development of diagnostic tools are funded by the UK Department for International Development ${ }^{13}$, the Foundation for Innovative New Diagnostics, Geneva, Switzerland (www.finddiagnostics.org) and the Doris Duke Charitable Foundation (www.ddcf.org). 
For optimal use in low-resource settings, it is also important for tests to be rapid, simple to use (that is, requiring little in the way of facilities, equipment or training), low-cost or cost-effective, easily interpretable, and stable when transported and stored under extreme conditions. For some infectious agents, tests are needed to better distinguish past from current infections. For others, improved POC methods are needed to return same-day test results so that patients can receive appropriate therapy while they are still at the clinic. Multiplexed tests are especially needed to accurately identify the aetiologic agent causing a disease that could have multiple causative agents, such as acute lower respiratory infections, diarrhoeal diseases, acute onset fevers and sexually transmitted infections. Global health programmes are significantly hindered because such tests are currently unavailable. There is also need for individual tests for emerging and re-emerging diseases such as tuberculosis, severe acute respiratory syndrome (SARS) and influenza, as well as vaccine-treatable diseases such as measles, tetanus and polio. For HIV/AIDS, better and more appropriate tests are needed for early diagnosis, case management and treatment monitoring of patients. For tropical diseases, the more urgent needs include those for leishmaniasis, trypanosomiasis and malaria case management and test-of-cure.

\section{Diagnostic tools used in developed-world centralized labs}

Microfluidic instrumentation can be and has been applied to several of the four most common centralized laboratory techniques - blood chemistries, immunoassays, nucleic-acid amplification tests and flow cytometry.

Basic blood chemistry panels consisting of 12 to 20 tests are routinely run on automated analysers to monitor a wide range of physiological functions. Analytes include blood enzymes, gases, electrolytes, lipids, thyroid indicators and drugs. Immunoassays in a wide range of formats allow quantification and monitoring of small molecules, large proteins and even whole pathogens. Simple and rapid immunoassays such as lateral flow strip tests (see below) can also be used in clinics or are available over the counter for home use. Blood, plasma, serum, urine, salivary fluids and other exudates are all used as samples. Nucleic-acid amplification tests, such as the polymerase chain reaction (PCR) and nucleic-acid sequence-based amplification, have been developed that can detect very small copy numbers of specific nucleic-acid sequences. Test kits are now commercially available for tuberculosis, HIV and sexually transmitted infections (STIs). Test sensitivity and specificity often exceed that of immunoassays and culture methods ${ }^{14}$. 'Real-time' PCR testing is now capable of producing a quantitative result in $20-30$ minutes $^{15,16}$. Flow cytometry is the method of choice for counting cells with specific physical and/or chemical characteristics. Great strides towards miniaturization, including work now published for a decade about microfluidic devices, have been made ${ }^{17,18}$.

These technologies are well suited to small and large laboratories in the developing world, assuming that the technicians employed to use them have a relatively high level of training. However, in places with lower levels of training, or away from venues with continuous power and, hence, refrigeration, these technologies have been less applicable. Microfluidics may be able to close the gap between what can be done now and what needs to be done at the remotest ends of the healthcare system, where the vast majority of the developing world's population resides.
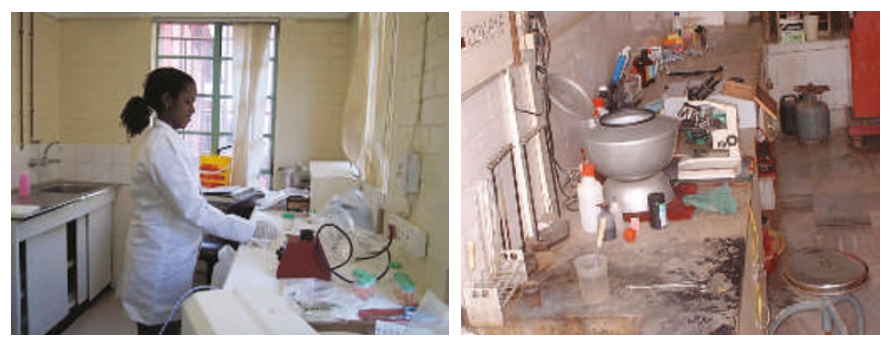

Figure 2 | Two typical laboratories in mid-level healthcare centres in the developing world. Note the wide range of conditions in which healthcare workers operate. (Images courtesy of B. H. W. and colleagues, PATH, USA.)
End-users of microfluidic diagnostics in the developing world The centralized laboratory model found in the developed world is not widely applicable in developing nations. Where such centralized laboratories exist at all, most are found in large cities, catering primarily for the affluent few. Healthcare facilities in rural areas commonly have only basic equipment, healthcare workers may have little training, and the resources to maintain complex equipment and handle fragile reagents are often limited. Three tiers of healthcare providers can be identified in developing countries: capital- or major-city-level hospitals; districtlevel healthcare providers (Fig. 2); and village-level healthcare workers. However, the actual level of care provided at each site can vary considerably.

Typical conditions in a laboratory in a mid-level healthcare centre in a developing country are rather different from those in a developed-world mid-level clinical laboratory. Running water and electricity may or may not be available, but power is at best intermittent with wide fluctuations in voltage. The ambient temperature may range from 10 to more than $40{ }^{\circ} \mathrm{C}$. Dust, wind and contaminating pathogens are very common. Potentially high-risk human samples, containing biosafety level (BSL)-2 and -3 pathogens are routinely handled with few precautions other than, perhaps, gloves. Maintaining and calibrating even moderately complex instruments still presents a challenge. However, it may be possible to perform microscopy, most lateral flow assays, some blood chemistry and cytology, and some enzyme-linked immunosorbent assays (ELISAs) in these labs.

At the periphery of the medical care system - in townships and villages - power, running water and refrigeration are often intermittent or absent. Whatever assays are to be performed must therefore be completely self-contained. Devices must be battery-operated, require no maintenance or calibration, be dust-proof, be easily secured at night, and operate at a wide range of ambient temperatures. Village-level healthcare workers are, for most of the population, the only or main source of healthcare; such health workers can sometimes perform basic strip or dipstick tests for pregnancy, HIV and, in some cases, malaria and STIs. Test results must be presented in a very simple, yes/no way to avoid misinterpretation. To bring a more sophisticated set of diagnostic capabilities to such environments is a challenge of both technical function and development of an appropriate user interface.

\section{Potential benefits of microfluidic diagnostic systems}

During the past decade in the developed world, near-patient testing using POC devices has become established for doctors' offices, stat labs and, in rare cases, in the home (for example, glucose monitors). Interest in moving to a more patient-centric POC/home-testing approach is on the rise in the developed world ${ }^{19}$. Microfluidic lab-on-a-chip technology may now suit both developing- and developed-world applications. Many of us believe we will soon have microfluidics-based POC and home-care devices that can perform assays at sensitivity, specificity and reproducibility levels similar to those of central laboratory analysers, but yet require little user input other than the insertion of the sample. Such devices could, in the hands of developing-country lay people, perform routine testing, or detect the presence of an infectious agent with epidemic potential such as influenza, an opportunistic infection, or a chronic health condition, and provide guidance to the end-user. That same diagnostic platform, loaded with different reagents, could, in the hands of a village healthcare worker, detect the aetiological agent causing acute fever or diarrhoea, and provide information on what, if any, therapy should be given to the patient. However, the cost of the hypothetical microfluidics-based diagnostic tool must be extremely low if it is to be applicable to the developing world.

Potential benefits of distributing diagnostic devices and systems designed specifically for developing countries include: access to diagnostic tools not previously available, and thus faster/more accurate diagnoses; better epidemiological data that can be used for disease modelling, vaccine introduction and to define the economics of a healthcare system; better utilization of minimally trained healthcare workers; and better use of existing therapeutics. 


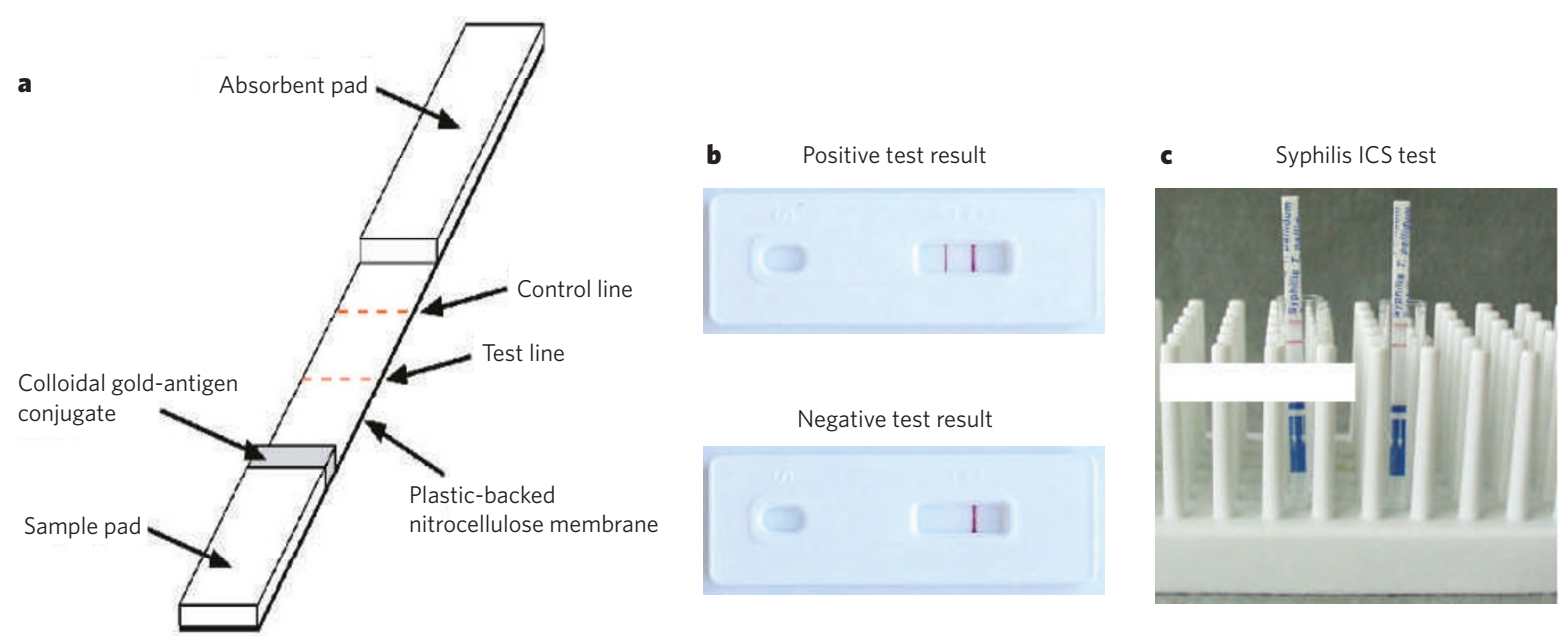

Figure 3 | Rapid immunochromatographic strip (ICS) tests for sexually transmitted infections. a, A schematic of the ICS assay format. b, c, Tests for gonorrhoea (b) and syphilis (c) are shown.

\section{Choices and challenges in the developing world}

There are three approaches to diagnostic technologies that have been used in the developed world: permanent integrated instruments, pure disposables and permanent instruments that use disposable components. All can be and have been adapted for use in the developing world, but they are not equally applicable to specific problems.

\section{Permanent integrated instruments}

The permanent integrated instrument is the mainstay of the centralized laboratory in the developed world. These systems are well suited to high-throughput work, but generally require an infrastructure that cannot usually be provided in developing countries. Even if such an instrument could be scaled down (and made affordable enough) to work in resource-limited settings, the requirement that the instrument purge itself of one sample before analysing another (preventing carryover) and that it be frequently calibrated with standards, and rinsed with cleaning solutions, is not in keeping with the setting. This approach is, therefore, unlikely to be successful, particularly in the most impoverished settings, even with microfluidic components.

\section{Disposables}

Lateral flow or immunochromatographic strip (ICS) tests have been, for the past decade, one of the very few diagnostic technologies to be successfully used in the developing world, and are, therefore, the technology that microfluidics must complement or supplant. They provide POC diagnosis in areas without access to well-equipped and well-staffed clinical laboratories. They rely on relatively inexpensive, off-the-shelf components and reagents, are amenable to large-scale production methods, and are relatively affordable. They can be formatted for detection of either antigens or antibodies, and are usable with a wide range of specimens. Most are stable at ambient temperatures for more than a year when appropriately packaged and can be shipped without refrigeration. The analytical performance of some POC lateral flow tests are comparable to reference-level laboratory methods ${ }^{3}$.

ICS tests developed by PATH, with support from the United States Agency for International Development (USAID) can diagnose diphtheria toxin ${ }^{20,21-27}$ and a number of STIs ${ }^{28}$, including gonorrhoea (Neisseria gonorrhoeae) (Fig. 3), syphilis (Treponema pallidum) ${ }^{29}$, chancroid (Haemophilus ducreyi) $^{20}$ and chlamydia (Chlamydia trachomatis) ${ }^{28,30-32}$. The early diagnosis offered by some strip tests, together with the ability to provide appropriate therapy to patients before they leave the clinic, has proved useful to control the spread of the diseases ${ }^{33}$. In addition to STI assays, PATH has developed, co-developed, or introduced assays for retinol-binding protein (as a marker for vitamin A deficiency) ${ }^{34}$, P. falciparum malaria ${ }^{30-32}, \mathrm{HIV}^{25,29}$, hepatitis $\mathrm{B}^{24}$, the hormone human chorionic gonadotropin (indicating pregnancy), faecal leukocytes and proteinuria $^{20-23,26,28}$.

Health workers can quickly learn to perform such ICS-based tests and require infrequent retraining. However, some such tests are still not sufficiently sensitive or specific for accurate POC use. For example, PATH's malaria test, which can only detect $P$. falciparum ${ }^{31}$, must be used with care in hyperendemic areas, where many individuals may have low titres of circulating $P$. falciparum antigens, leading to an excess of false positive results. Parasite antigens may persist in circulation even after successful therapy, and could produce confusing results with $P$. falciparum tests when assessing test-of-cure or drug resistance. The visual readout of the strip is usually limited to a yes/no answer; this is not adequate when the level of an analyte is important. Sophisticated sample preconditioning (which is needed for many assays) is also impossible in many areas.

\section{Disposables with a reader}

The compromise that allows high performance with low per-test cost, but with added complexity, is to use a 'reader' with single-use disposables. The sample and waste are retained within the disposable, so the reader does not need to be cleaned between samples. Calibrants can also be stored on the disposable. At the University of Washington, we are developing a system for monitoring analytes in saliva using surface plasmon resonance (SPR) imaging ${ }^{35-37}$. The aim of the salivary diagnostics system is to measure small-molecule analytes such as hormones and drugs in whole saliva. The use of saliva is a suitably challenging problem, and one that would translate well to use in the developing world. The 'disposables with a reader' model seems to us to be the most promising approach as a powerful and versatile format that can meet the demands of the developing-world setting. Below, we consider in more detail the challenges to overcome for this diagnostic platform.

The first challenge is to process complex biological samples without the sophisticated sample preconditioning capabilities (human or mechanical) available in centralized labs. Centrifugation, which might be available in a mid-level laboratory to provide a purified plasma sample from blood, will not be available in a remote setting. There are uniquely microfluidic solutions to these issues, such as the H-filter ${ }^{38-41}$, which was conceived as an alternative to a conventional porous barrier filter (Fig. 4). The H-filter can be used to limit the size of analytes that proceed downstream to a detection module from non-blood samples such as saliva, at the cost of requiring controlled flow to three of the four ports of the device. However, for single-use disposables, a conventional filter may be adequate for removing cellular components from blood prior to analysis. In the case of the salivary diagnostics system, it was found that saliva contained molecules that rapidly bound to the SPR detection surface, adding unacceptable background signal, so 


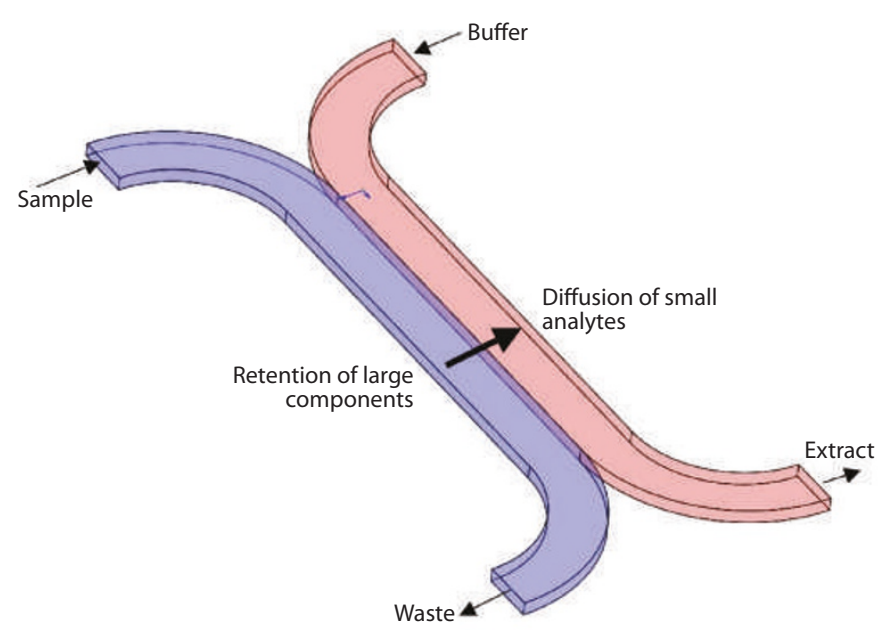

Figure 4 | Schematic of an H-filter. This is a microfluidic device that allows convenient extraction of small molecules from complex fluids into simpler buffer streams. Initial attempts to use the $\mathrm{H}$-filter alone to extract smallmolecule analytes from whole saliva failed because of the high viscosity and non-newtonian behaviour of the solution. It was therefore necessary that the concentration of mucins be reduced before the saliva entered the H-filter (manuscript in preparation, K.H., B. Finlayson and P.Y.). Note also that in the disposable card in Fig. 5, the $\mathrm{H}$-filter channels were configured to allow greater contact between the two flowing streams.

some form of sample preconditioning was necessary. Electrophoresis, dielectrophoresis and isoelectric focusing are three other techniques that can also be used, and have been used extensively in lab-on-a-chip systems $^{42-51}$. The electrokinetic methods can add cost if they require electrodes to be present in the disposable itself.

One of the great strengths of microfluidics is the ability to integrate the steps of a complex chemical process into a monolithic disposable - an assay performed in such a way can be more precise, more accurate and more reproducible than the same assay performed by hand. An example of an integrated assay is shown in Fig. 5, in which many of the steps of analysis of a whole saliva sample (subsequent to mechanical filtration) are integrated into a disposable diagnostic card. In this card, the sample is isolated and the waste is retained, and sample contact with the electrical and optical components of the permanent instrument is avoided. Note that not all of the challenges of integration have been accomplished in the current design; for example, external pumps and off-chip sample preprocessing are required. However, two other projects that are underway (and described briefly below) will produce disposables that fully integrate all fluidic functions.

The selection of a material for use in a disposable must balance the inherent cost of the material with processing costs. Many polymers are inherently inexpensive, but the cost of processing to make them compatible with device function can add substantially to the total cost of the disposable. For example, microfluidic materials must be selected to minimize surface adsorption of analytes and to allow those analytes to move to the detection zones in the disposables. A material can be inherently nonfouling, but more often must undergo a coating process to be made so. An inherently low-cost material may be more difficult to chemically modify and would increase the complexity of manufacturing and the final cost of the disposable.

Often, selection of a material will be dictated by application requirements. For example, in applications that require fluorescence-based detection of small-molecule analytes, low fluorescence (and low light scattering) is a selection criterion for acceptable device materials in the optical path ${ }^{52}$. One material, poly(dimethylsiloxane) (PDMS), is excellent for fabrication of low-fluorescence devices, but is permeable to many small and hydrophobic molecules, and is not readily or economically formed in high-throughput production. Thermoplastics such as polystyrene are ideal for high-level production because they can be injection molded, although not all have optimal optical properties. Whereas lamination of laser-cut polymer sheets is extremely cost-effective for rapid prototyping ${ }^{53}$, it is not nearly as economical as injection moulding for production of large numbers of devices. Although mixing different materials is complex, it may often be necessary. Note that in the laminated disposable example (Fig. 5a), PDMS was used to form a herringbone mixer ${ }^{54}$ with small features, largely because the $\mathrm{CO}_{2}$ laser (used to form the Mylar layers) had too low a spot resolution. Also, the gold SPR imaging surface was formed on a glass microscope slide because it was less expensive to manufacture them this way than to purchase small lots of gold films of a precise thickness on polymers. The fragility of glass makes it unsuitable for use in a commercializable disposable for POC diagnostics. Real devices will always have to combine multiple materials.

Because one of the fundamental requirements for a microfluidic diagnostic disposable is extremely low cost (ideally pennies), the disposables must be designed to have few parts and be inexpensive to manufacture, ideally using injection moulding whenever possible. These criteria rule

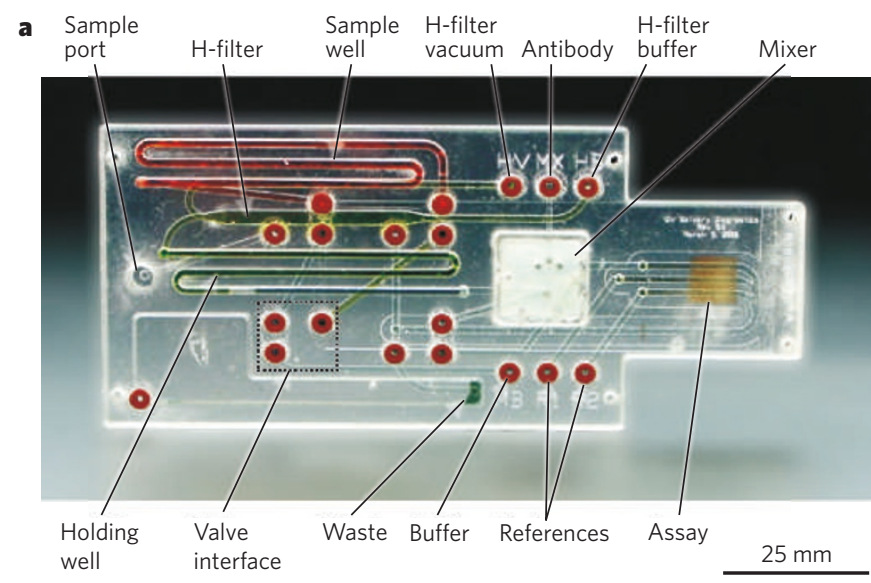

b

Sample syringe Syringe filter

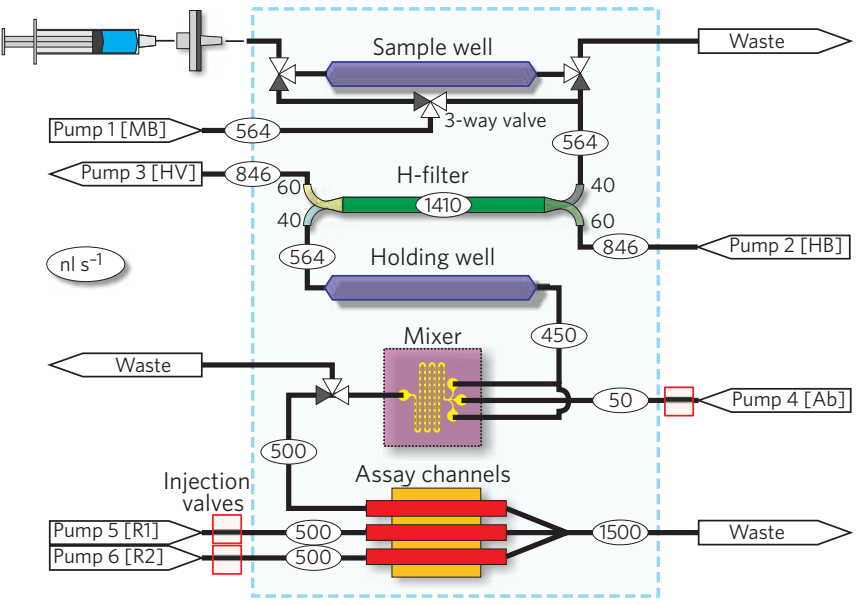

Disposable card and off-card manifold with valves

Figure 5 | Example of an integrated disposable diagnostic card. a, Image of a card. The red $\mathrm{O}$-rings are for interfacing with off-card components (for example, valves and pumps) that will eventually be incorporated onto the card itself. b, Schematic of the card. The card accepts filtered saliva (see Table 2) from the syringe and contains an $\mathrm{H}$-filter for further sample conditioning, a herringbone mixer for mixing antibodies with the sample, and channels with gold-coated surfaces for detection of analyte in the sample using an SPR imaging-based immunoassay (manuscript in preparation, T.E. et al.). Numbers in ovals are flow rates in $\mathrm{nl} \mathrm{s}^{-1}$. Numbers at the $\mathrm{H}$-filter's four ports are the percentages of flow entering (right) and exiting (left) the device. MB, mixing buffer; HB, H-filter buffer; HV, H-filter vacuum; R1 and $\mathrm{R} 2$ are reference solutions, typically a positive and negative control. 
a

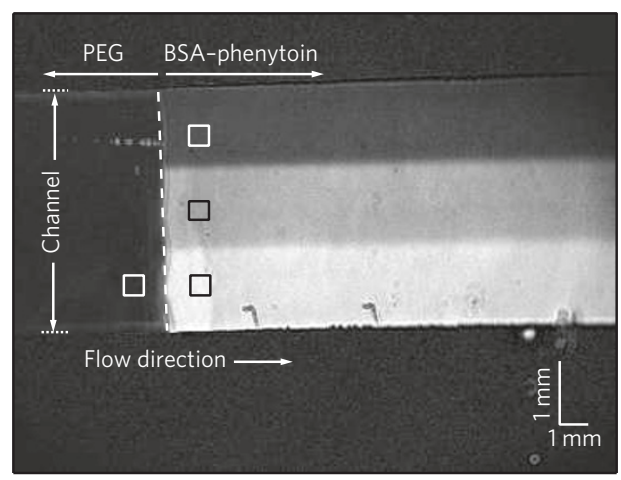

b

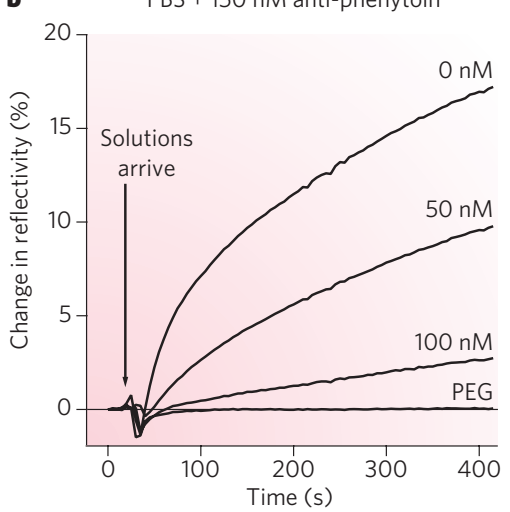

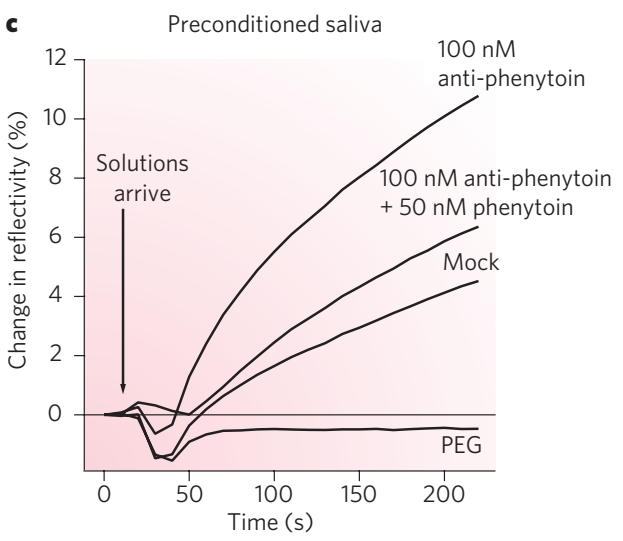

Figure 6 | Quantification of a competitive immunoassay for phenytoin using surface plasmon resonance (SPR). a, SPR difference image used to quantify a competitive immunoassay for phenytoin (manuscript in preparation, K.N. et al.). The detection zone is a gold surface precoated with bovine serum albumin (BSA) covalently modified by phenytoin. The three streams $\left(750 \mathrm{nl} \mathrm{s}^{-1}\right)$ contained $150 \mathrm{nM}$ anti-phenytoin antibody mixed with $0 \mathrm{nM}, 50 \mathrm{nM}$ or $100 \mathrm{nM}$ soluble phenytoin (bottom to top, respectively). The contrast in the image has been adjusted to highlight the differences between the nonfouling polyethylene glycol (PEG) upstream and the three sample regions. b, c, Competition assay detection of low-end therapeutic levels of phenytoin in a model system (b) and in preconditioned saliva (c). b, SPR reflectivity over time of anti-phenytion antibody in phosphate buffer binding to a BSA-phenytoin-treated surface. The plot shows that the rate of antibody binding negatively correlates with the amount of competitor (phenytoin) added to the solution. c, SPR reflectivity of variously treated preconditioned saliva samples over time. In this case, whole human saliva was preconditioned using a mechanical filter and the $\mathrm{H}$-filter offcard. Plots $\mathbf{b}$ and $\mathbf{c}$ show that the PEG region effectively resisted fouling to either antibody or components present in preconditioned saliva.

out inherently expensive materials, complex manufacturing methods and expensive reagents in anything but the smallest quantities. It is also advisable, on cost grounds alone, to assemble and manufacture as many parts of the disposable as possible in the developing world.

The disposable/reader model results in the creation of biohazardous medical waste of little or no value for recycling. The best method for disposing of such waste is incineration, but it is also possible to render it non-infectious by treatment with a disinfectant such as bleach, which could even be incorporated into the disposable in a blister pack. For use in remote areas, thought should therefore be given to producing an efficient, safe and verifiable method for incinerating disposables using local facilities.

The microfluidic diagnostic end-users will need a supply of disposables. The disposables will need to be robust, as they will be transported at ambient temperatures. Beacause the disposables will be hand-carried, their weight must be minimal. Large, centralized instruments frequently require large volumes of pre-made buffers and reagent solutions to operate. Fluid reservoirs contribute substantially to the bulk of the instrumentation (and the revenue stream of the manufacturers). Because water is heavy, there is a clear incentive to ship the disposables dry, and use local sources of purified water.

Although lateral flow strips and related technologies do not require power to operate, instruments currently used in centralized laboratories are designed with no consideration of their electrical requirements. For a field-portable instrument, the assumption is that electrical power will either be nonexistent, or limited to automobile generators, photocells, hand-generators, or other low-capacity power sources. As a consequence, MDT systems must be designed from the ground up to use a minimum amount of power, and if the disposables store power, it must be in clever ways - a challenging task for any but the simplest (and least quantitative) assays. Such high-precision assays - for example, those requiring thermal cycling (such as PCR and related nucleic-acid amplification technologies) - require a lot of power in their current embodiments, so there is room for creative engineering to develop inherently low-power methods of changing sample temperature. What power sources may be used (other than external ones) must be compact and lightweight. Great advances made during the last few years in small, rechargeable batteries will help, but there is clearly scope for improvement in this area too.

A successful microfluidic diagnostic technology will be self-calibrating, carrying all necessary reagents to run positive and negative controls along with it. This is particularly necessary for a POC instrument, which will be especially sensitive to variations in ambient temperature because of its small size. Ideally, these controls will be present on the disposable itself, so that every measurement will be made at the same time as the calibration runs. This will put a heavy burden on the preservation of the reagents in the disposable. In the salivary diagnostics project, we have developed microfluidic methods for running calibrations alongside the critical measurements by patterning reagents and using multiple lowReynolds-number fluid streams to keep detection areas discrete. Such methods allow measurement of binding rates of analytes to surfaces in samples such as saliva in just a few minutes (Fig. 6).

The disposables themselves will be shipped and stored at ambient temperatures, which, in the developing world, can range from tropical to arctic. For vaccines, which are generally very heat-labile, the 'cold chain' has been extended to the periphery of the healthcare system. However, it is costly to maintain. The disposables for diagnostics must contain biological molecules (and perhaps even entire pathogens) in addition to a range of organic chemicals as reagents. If they were stored in buffer at ambient temperature they would very rapidly be degraded. A preferred alternative, successfully used in lateral flow assays and recently applied to more readily identifiable 'microfluidic' systems, is to desiccate the biomolecules, replacing the normal waters of hydration by sugars ${ }^{55,56}$. This, derived in part from the drug-delivery field, would allow the disposable to contain only dry reagents, thus reducing weight, extending product stability and allowing transportation and storage of the MDTs at ambient temperature.

A critical aim of microfluidic diagnostics for the developing world is to make all steps of their use simple and as culturally independent as possible. This will be a challenge given the diversity of language, training and cultural backgrounds of the end-users of the technologies. For this reason, extensive work with the end-users is an absolute requirement before designing the user interface. It is not possible to assume that the end-user will be the same as the users of centralized laboratory equipment.

\section{The future of MDTs in the developing world}

Enteric infections are the second leading cause of morbidity and mortality worldwide, accounting for an estimated 3.1 million deaths annually, mostly in the developing world. Outbreaks can be controlled with rapid diagnosis and appropriate treatment, which can also reduce the severity of disease. But the developed-world standards for diagnosis of infectious diarrhoea - culture, enzyme immunoassay and PCR - are impractical, expensive and too slow for developing-world users. Identification of target pathogens, even in the best developed-world laboratory, often takes 2-4 days ${ }^{57}$. 
Table 1 | Factors that will affect the use of a microfluidic diagnostic technology (MDT) and the attributes of a successful MDT in the developing world

\begin{tabular}{ll}
\hline Key factors that will affect the introduction, acceptance and sustained use of an MDT & Key attributes of an MDT \\
\hline Cost of the technology & Low cost \\
\hline Degree of accuracy & High degree of accuracy (for example, low rate of false positives) \\
\hline Quality control & Reproducible chip performance (using on-chip calibrators) \\
\hline Level of training of users & User interface that requires little training \\
\hline Length of time to obtain test result & Short time to test result \\
\hline Performance in a variety of settings & Stable ambient temperature storage and low power consumption \\
\hline Performance under variable operating conditions (such as temperature or humidity) over time & Reproducible operation in variable environments, and ruggedness \\
\hline Local education on health issues & A high perceived need for the test \\
\hline Availability of successful therapies & Potential for significant health improvements \\
\hline
\end{tabular}

Work has begun on a 'disposable with reader' lab-on-a-chip platform for identification of the pathogens - Shigella dysenteriae type 1 , Shiga toxin-producing Eschcherichia coli (O157:H7), Campylobacter jejuni and Salmonella - that commonly cause acute enteric disease with similar symptoms. The diagnostic assay comprises the disposable single-use microfluidic card, containing dry, heat-stable agents and a permanent hand-held instrument to operate the microfluidic circuits and control the card's temperature. The user will insert a swab containing a stool sample into the card and place the card in the instrument. The card will include four microfluidic subcircuits: organism capture and lysis from raw stool; nucleic-acid capture; multiplexed nucleicacid amplification; and visual detection of amplified PCR products. A combination of capillary action and positive displacement pumping will draw the sample via microfluidic channels through the integrated subcircuits on the disposable. A positive control (E. coli, present in any stool sample) will be included to demonstrate proper sample processing, and to validate negative results. The complete sequence will take less than $30 \mathrm{~min}$. Early tests show that sensitivity and specificity are comparable to the results achieved with conventional microbiological and PCR assays ${ }^{58}$, and the cost is expected to be between US $\$ 1$ and $\$ 5$ per disposable.

The Gates Foundation's Grand Challenges in Global Health initiative is supporting the development of prototypes of a disposable/ hand-held reader system, which will include the reagents for both immunoassays and nucleic-acid amplification tests, requiring only a couple of drops of blood from the patient for a readout. Initial focus is on the development of tests for simultaneous detection of infectious agents that cause diseases associated with rapid onset fever, including malaria, typhoid, dengue, rickettsial diseases, measles and influenza. Because of the ability to perform both immunoassays and nucleic-acid amplified tests, this platform could ultimately process a wide range of disease panels.

The current funding climate for the development of diagnostics for developing countries is good, thanks to vastly increased interest in global health, stoked by initiatives from private foundations and institutions. Additionally, significant funding of pathogen diagnostics development is available through United States government sources ${ }^{12}$, although primarily driven by biodefence concerns, as many of the organisms that could be weaponized also cause disease in developing countries (www3.niaid.nih.gov/biodefense/bandc_priority.htp). Donors and international agencies support the need for new and improved diagnostic tools for priority diseases; advocacy and technology development groups have been formed, such as the STD Diagnostics Initiative ${ }^{59}$ and the Tuberculosis Diagnostics Initiative ${ }^{60}$, with secretariats within the World Health Organisation/Special Programme for Research and Training

Table 2 | Effectiveness of preconditioning methods for salivary diagnostics

\begin{tabular}{llll} 
Sample & Whole saliva & Filtrate & Extract \\
\hline Mucin/glycoprotein & $100 \%$ & $27 \%$ & $2 \%$ \\
\hline Total protein & $100 \%$ & $55 \%$ & $9 \%$ \\
\hline Analyte (cortisol) & $100 \%$ & $92 \%$ & $27 \%$
\end{tabular}

Mucin, total protein and analyte content for whole human saliva, the filtrate (after the use of the depth filter) and the extract (after extraction with the $\mathrm{H}$-filter). in Tropical Diseases (WHO/TDR). These are good beginnings, but because the marketplace for medical diagnostics is fragmented in the developing world, no single strategy will ensure distribution of new technologies as they are developed.

Although we feel strongly that the development of appropriate diagnostic technologies is an important factor in the goal of improving global public heath, significant improvements in the health of the developing world will only be achieved if there is tight coordination between the diagnostics developers and the communities involved in local education, drug discovery and drug distribution. Long-term, successful use of MDTs in the developing world will require the sustained efforts of these communities.

There are a number of key factors that will affect the introduction, acceptability and sustainability of these technologies (summarized in Table 1). One of the greater challenges in deploying microfluidic diagnostic systems in the developing world will be bringing the cost down close to the cost of the most inexpensive of current tests, namely the lateral flow immunoassays (it should be borne in mind that the actual cost of using these immunoassays does include that of misdiagnosing patients). Thanks to increased interest on the part of the global health community, we expect to see the introduction of microfluidic diagnostic devices specifically designed for the developing world within the next 5 years.

1. Dupuy, A., Lehmann, S. \& Cristol, J. Protein biochip systems for the clinical laboratory. Clin. Chem. Lab. Med. 43, 1291-1302 (2005).

2. Toner, M. \& Irimia, D. Blood-on-a-chip. Annu. Rev. Biomed. Eng. 7, 77-103 (2005).

3. von Lode, P. Point-of-care immunotesting: approaching the analytical performance of central laboratory methods. Clin. Biochem. 38, 591-606 (2005).

4. World Bank World Development Report 1993: Investing in Health (Oxford Univ. Press, New York, 1993)

5. Black, R. E., Morris, S. S. \& Bryce, J. Where and why are 10 million children dying every year? Lancet 361, 2226-2234 (2003).

6. World Health Organization World Health Report 2002 - Reducing Risks, Promoting Healthy Life [online] <www.who.int/whr/2002/en/index.html> (2002).

7. Morens, D. M., Folkers, G. K. \& Fauci, A. S. The challenge of emerging and re-emerging infectious diseases. Nature 430, 242-249 (2004)

8. Fauci, A. S. Infectious diseases: considerations for the 21st century. Clin. Infect. Dis. 32, 675-685 (2001)

9. National Research Council Committee on Science and Technology in Foreign Assistance The Fundamental Role of Science and Technology in International Development: An Imperative for the US Agency for International Development (Washington DC, 2006).

10. Becker, G. S., Philipson, T. J. \& Soares, R. R. The quantity and quality of life and the evolution of world inequality. Am. Econ. Rev. 95, 277-291(2005).

11. Murray, C. J. L. \& Lopez, A. D. The Global Burden of Disease: a comprehensive assessment of mortality and disability from diseases, injuries, and risk factors in 1990 and projected to 2020 (Harvard Univ. Press, Cambridge, Massachusetts, 1996).

12. Conroy, J. Developing biodefense IVDs still a priority. IVD Technol. 12, 37-46 (2006).

13. UK Department for International Development The Challenge of TB and Malaria Control [online] <http://www.dfid.gov.uk/pubs/files/tb-malaria-control.pdf> (2005).

14. Jalal, H., Stephen, H., Al-Suwaine, A., Sonnex, C. \& Carne, C. The superiority of polymerase chain reaction over an amplified enzyme immunoassay for the detection of genital chlamydial infections. Sex. Transm. Infect. 82, 37-40 (2006)

15. Boel, C. H., van Herk, C. M., Berretty, P. J., Onland, G. H. \& van den Brule, A. J. Evaluation of conventional and real-time PCR assays using two targets for confirmation of results of the COBAS AMPLICOR Chlamydia trachomatis/Neisseria gonorrhoeae test for detection of Neisseria gonorrhoeae in clinical samples. J. Clin. Microbiol. 43, 2231-2235 (2005).

16. Eickhoff, M. et al. Ultra-rapid detection of Chlamydia trachomatis by real-time PCR in the LightCycler using SYBR green technology or 5'-nuclease probes. Clin. Lab. 49, 217-225 (2003).

17. Dittrich, P. S. \& Manz, A. Single-molecule fluorescence detection in microfluidic channels-the Holy Grail in $\mu$ TAS? Anal. Bioanal. Chem. 382, 1771-1782 (2005). 
18. Rodriguez, W. R. et al. A microchip CD4 counting method for HIV monitoring in resource-poor settings. PLoS Med. 2, e182 (2005).

19. Price, C. P. Regular review: point of care testing. Brit. Med. J. 332, 1285-1288 (2001).

20. Patterson, K. et al. Development of a rapid immunodiagnostic test for Haemophilus ducreyi. J. Clin. Microbiol. 40, 3694-3702 (2002).

21. Engler, K. H. et al. Immunochromatographic strip test for rapid detection of diphtheria toxin: description and multicenter evaluation in areas of low and high prevalence of diphtheria. J. Clin. Microbiol. 40, 80-83 ( 2002).

22. Zarakolu, P., Buchanan, I., Tam, M., Smith, K. \& Hook, E. W. Preliminary evaluation of an immunochromatographic strip test for specific Treponema pallidum antibodies. J. Clin. Microbiol. 40, 3064-3065 (2002)

23. Talbot, E. A. et al. Tuberculosis serodiagnosis in a predominantly HIV-infected population of hospitalized patients with cough, Botswana, 2002. Clin. Infect. Dis. 39, 1-7 (2004).

24. Raj, A. A., Subramaniam, T., Raghuraman, S. \& Abraham, P. Evaluation of an indigenously manufactured rapid immunochromatographic test for detection of $\mathrm{HBsAg}$. Indian J. Pathol. Microbiol. 44, 413-414 (2001).

25. Kaur, H., Dhanao, J. \& Oberoi, A. Evaluation of rapid kits for detection of HIV, HBsAg and HCV infections. Indian J. Med. Sci. 54, 432-434 (2000).

26. Parkes, R., Lo, T., Wong, Q., Isaac-Renton, J. L. \& Byrne, S. K. Comparison of a nested polymerase chain reaction-restriction fragment length polymorphism method, the PATH antigen detection method, and microscopy for the detection and identification of malaria parasites. Can. J. Microbiol. 47, 903-907 (2001).

27. Cates, W. Preserving fertility: an underappreciated aspect of sexual health. Network $\mathbf{2 3}$ no. 2 (2003).

28. Tam, M. R. in Sexually Transmitted Diseases (eds Holmes, K. K. et al.) 1409-1420 (McGraw-Hill, New York, 1999).

29. Cayemittes, M., Hankins, C. \& Tam, M. R. An AIDS test that travels well. IDRC Rep. 21, 27-28 (1993)

30. Loutfy, M. R., Assmar, M., Hay Burgess, D. C. \& Kain, K. C. Effects of viral hemorrhagic fever inactivation methods on the performance of rapid diagnostic tests for Plasmodium falciparum. J. Infect. Dis. 178, 1852-1855 (1998).

31. Mills, C. D., Hay Burgess, D. C., Taylor, H. J. \& Kain, K. C. Evaluation of a rapid and inexpensive dipstick assay for the diagnosis of Plasmodium falciparum malaria. Bull. World Health Organ. 77, 553-559 (1999).

32. Labbe, A. C. et al. The performance and utility of rapid diagnostic assays for Plasmodium falciparum malaria in a field setting in the Lao People's Democratic Republic. Ann. Trop. Med. Parasitol. 95, 671-677 (2001)

33. Vickerman, P., Peeling, R. W., Watts, C. \& Mabey, D. Detection of gonococcal infection: pros and cons of a rapid test. Mol. Diagn. 9, 175-179 (2005).

34. Hix, J. et al. Development of a rapid enzyme immunoassay for the detection of retinolbinding protein. Am. J. Clin. Nutr. 79, 93-98 (2004).

35. Chinowsky, T. et al. Compact surface plasmon resonance imaging system for saliva-based medical diagnostics. Biosens. Bioelectron. (submitted)

36. Fu, E., Chinowsky, T., Foley, J., Weinstein, J. \& Yager, P. Characterization of a wavelengthtunable surface plasmon resonance microscope. Rev. Sci. Instrum. 75, 2300-2304 (2004).

37. Fu, E., Foley, J. \& Yager, P. Wavelength-tunable surface plasmon resonance microscope. Rev. Sci. Instrum. 74, 3182-3184 (2003)

38. Yager, P., Holl, M. R., Weigl, B. H. \& Brody, J. P. (Univ. Washington, 1998).

39. Brody, J. P. \& Yager, P. Diffusion-based extraction in a microfabricated device. Sens. Actuators A Phys. A58, 13-18 (1997).

40. Brody, J. P., Yager, P., Goldstein, R. E. \& Austin, R. H. Biotechnology at low Reynolds numbers. Biophys. J. 71, 3430-3441 (1996).

41. Brody, J. P., Osborn, T. D., Forster, F. K. \& Yager, P. A planar microfabricated fluid filter. Sens. Actuators A Phys. 704-708 (1996).

42. Gascoyne, P. R. C. \& Vykoukal, J. Particle separation by dielectrophoresis. Electrophoresis 23, 1973-1983 (2002)
43. Gascoyne, P. et al. Microsample preparation by dielectrophoresis: isolation of malaria. Lab Chip 2, 70-75 (2002)

44. Wang, X. B. et al. Cell separation by dielectrophoretic field-flow-fractionation. Analyt. Chem. 72, 832-839 (2000).

45. Müller, T., Schnelle, T., Gradl, G., Shirley, S. G. \& Fuhr, G. Microdevice for cell and particle separation using dielectrophoretic field-flow fractionation. J. Liq. Chromatogr. Relat. Technol. 23, 47-59 (2000).

46. Hughes, M. AC electrokinetics applications for nanotechnology. Nanotechnology 11, 124-132 (2000).

47. Rousselet, J., Markx, G. H. \& Pethig, R. Separation of erythrocytes and latex beads by dielectrophoretic levitation and hyperlayer field-flow fractionation. Colloids Surf. A 140, 209-216 (1998).

48. Cabrera, C. R., Macounová, K., Holl, M. R. \& Yager, P. in IEEE 1st Annual Conference on Microtechnology in Medicine and Biology (ed. Beebe) (IEEE Press, Lyon, 2000).

49. Cabrera, C. R. \& Yager, P. Continuous concentration of bacteria in a microfluidic flow cell using electrokinetic techniques. Electrophoresis 22, 355-362 (2001).

50. Macounová, K., Cabrera, C. R. \& Yager, P. Concentration and separation of proteins in microfluidic channels on the basis of transverse IEF. Analyt. Chem. 73, 1627-1633 (2001).

51. Cheng, S. B. et al. Development of a multichannel microfluidic analysis system employing affinity capillary electrophoresis for immunoassay. Analyt. Chem. 73, 1472-1479 (2001).

52. Hawkins, K. R. \& Yager, P. Nonlinear decrease of background fluorescence in polymer thinfilms - a survey of materials and how they can complicate fluorescence detection in $\mu$ TAS Lab Chip 3, 248-252 (2003).

53. Yager, P. et al. in Micro Total Analysis Systems ' 98 (eds Harrison, D. J. \& van den Berg, A.) 207-212 (Kluwer Academic, Dordrecht, Banff, 1998).

54. Stroock, A. et al. Chaotic mixer for microchannels. Science 295, 647-651 (2002)

55. Garcia, E., Kusmanto, F., Finlayson, B. \& Yager, P. in Micro Total Analysis Systems 2003 (eds Northrup, M. A., Jensen, K. F. \& Harrison, D. J.) 551-554 (Mesa Monographs, Enschede, 2003)

56. Garcia, E., Kirkham, J. R., Hatch, A. V., Hawkins, K. R. \& Yager, P. Controlled microfluidic reconstitution of functional protein from an anhydrous storage depot. Lab Chip 4, 78-82 (2004).

57. Guerrant, R. L. et al. Practice guidelines for the management of infectious diarrhea. Clin. Infect. Dis. 32, 331-351 (2001).

58. Weigl, B. et al. Fully integrated multiplexed lab-on-a-card assay for enteric pathogens. Proc SPIE 6112, 1-11 (2006)

59. Tam, M. R. \& Holmes, K. K. The STD diagnostic network: priorities for diagnosis of STDs in resource-poor settings. Ups. J. Med. Sci. Suppl. 50, 22-23 (1991).

60. Perkins, M. D. \& Kritski, A. L. Diagnostic testing in the control of tuberculosis. Bull. World Health Organ. 80, 512-513 (2002)

Acknowledgements We thank our colleagues at PATH, Micronics, Nanogen, Invetech, the University of Washington, Seattle, and Washington University in St Louis, Missouri, for their many contributions to ongoing and past projects, and our funding sources, currently including the National Institutes of Health (the National Institute of Allergy and Infectious Diseases, and the National Institute of Dental and Craniofacial Research) and the Bill \& Melinda Gates Foundation. Thanks to colleagues at PATH for the photographs of laboratories in the developing world.

Author Information Reprints and permission information is available at npg.nature.com/reprintsandpermissions. The authors declare competing financial interests: details accompany the paper at www.nature.com/nature. Correspondence should be addressed to P.Y. (yagerp@u.washington.edu). 